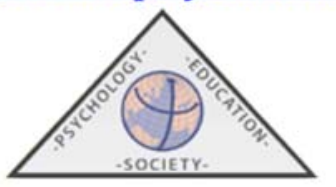

ISSN 2171-2085 (print) / ISSN 1989-709X (online)

\title{
La implementación de contenidos sobre diseño universal en ingeniería informática y arquitectura en España
}

\author{
Sergio SÁNCHEZ-FUENTES* y Emiliano DÍEZ-VILLORIA** \\ Universidad de Burgos y Universidad de Salamanca, España
}

(Received on March 1, 2015; Accepted on September 21, 2015)

\begin{abstract}
RESUMEN: En base a la legislación vigente, y por tanto, a la obligación que las universidades españolas tienen de enseñar contenidos relacionados con el diseño universal o diseño para todos en aquellas titulaciones que se relacionen con la participación de todos los ciudadanos en la sociedad, este trabajo tiene como objetivo fundamental hacer un análisis de cómo se está llevando a cabo este proceso en las titulaciones de ingeniería informática y arquitectura. Para ello, se ha contado con una muestra total de 159 guías docentes de asignaturas de ambas titulaciones de todas las universidades españolas. Los principales resultados del estudio demuestran que menos de la mitad de universidades estaría enseñando contenidos relacionados con el diseño universal y que existen un total de 59 titulaciones en las que se imparten contenidos relacionados con el diseño universal, aunque con diferencias significativas entre arquitectura e ingeniería informática. Finalmente, los autores discuten sobre la necesidad de cumplir la legislación vigente universitaria en la inclusión de contenidos relacionados con el diseño para todas las personas.
\end{abstract}

Palabras clave: Accesibilidad Universal, Diseño para todos, Diseño inclusivo, Universidad, Discapacidad.

The implementation of universal design content on computer engineering and architecture in Spain

\begin{abstract}
Current education legislation in Spain ensures the participation of all citizens in society consequently, universities have to teach about universal design. This study focuses on the implementation of this process in the undergraduate degrees of computer engineering and architecture. A total sample of 159 academic guides from both degrees werea nalysed. The main results of the study show that less than half of the universities teach universal design and there are 59 degrees in which universal design is taught, albeit with significant differences between architecture and computer engineering. Finally, the authors discuss the need to comply with university legislation on including design-related content for everyone.
\end{abstract}

Keywords: Universal Accessibility, Design for all, Inclusive Design, University, Disability.

Correspondencia: Sergio Sánchez. Departamento de Ciencias de la Educación. Facultad de Educación. C/ Villadiego s/n 09001 (Burgos), España. E-mail: ssfuentes@ubu.es.

\section{Introducción}

Con la llegada del Espacio Europeo de Educación Superior, las enseñanzas universitarias han visto modificadas las características y condiciones en las que se lleva a cabo la formación superior. Esto, añadido a la diversidad cada vez mayor que existe entre la 
población estudiantil universitaria, supone un reto para las demandas de la sociedad actual. Con el fin de comprender e incluir la amplia variedad posible de grupos de usuarios, las enseñanzas oficiales de grado en España deberían ofertar materias que incluyan contenidos sobre diseño universal, igualdad de oportunidades, accesibilidad, infoaccesibilidad o diseño para todos (Preiser, 2011).

Más concretamente, existen ciertas titulaciones que presentan una estrecha relación con los principios del diseño universal debido al origen de este concepto hace cuatro décadas. Por ello, titulaciones del área de la arquitectura o la ingeniería informática son consideradas por los autores como dos ámbitos de especial relevancia para la implantación de contenidos sobre diseño universal y, son por tanto, las áreas seleccionadas para llevar a cabo este estudio.

Esta situación, de hecho, es de obligado cumplimiento debido a las diferentes leyes que avalan los derechos de las personas con discapacidad (PcD de ahora en adelante) en la participación en la sociedad. La ley fundamental por la que las PcDtienen garantizados sus derechos, ya hace referencia a la accesibilidad y diseño para todos desde su propio título. Ésta es la Ley 51/2003, de 2 de diciembre, de igualdad de oportunidades, no discriminación y accesibilidad universal de las personas con discapacidad, más conocida como LIONDAU. Dicha ley, en su Artículo 10. Condiciones básicas de accesibilidad y no discriminación, se hace referencia a la accesibilidad, ya describe la necesidad de que las administraciones públicas cumplan con los requisitos establecidos por el diseño universal en sus instalaciones:

“2. Las condiciones básicas de accesibilidad y no discriminación establecerán,... medidas concretas para prevenir o suprimir discriminaciones, y para compensar desventajas o dificultades. Se incluirán disposiciones sobre, al menos, los siguientes aspectos:

a) Exigencias de accesibilidad de los edificios y entornos, de los instrumentos, equipos y tecnologías, y de los bienes y productos utilizados en el sector o área.”

Si bien es cierto que esto no supone una relación directa con el ámbito universitario, sí sirve para sentar las bases de aquellos conocimientos y competencias necesarias que se deberán adquirir en las enseñanzas superiores.

En lo que se refiere al ámbito educativo y su relación con la accesibilidad en España, la Ley Orgánica 2/2006, de 3 de mayo, de Educación, es la ley de carácter general aplicable a todas las enseñanzas regladas en España. Ésta recoge los principios básicos en materia de acceso a la educación por parte de los estudiantes con discapacidad. De la misma manera, la Ley Orgánica 4/2007, de 12 de abril, por la que se modifica la Ley Orgánica 6/2001, de 21 de diciembre, de Universidades, hace referencia en su Disposición adicional vigésima cuarta, a la necesidad de incluir contenidos relacionados con el diseño universal. Así, dicha ley expone lo siguiente:

“5. Todos los planes de estudios propuestos por las universidades deben tener en cuenta que la formación en cualquier actividad profesional debe realizarse desde el respeto y la promoción de los Derechos Humanos y los principios de accesibilidad universal y diseño para todos.”

Para concluir en lo referido al marco legal general que tiene relación con el diseño para todos o el diseño universal, habría que mencionar lo dispuesto en el Real Decreto 1393/2007, de 29 de octubre, por el que se establece la ordenación de las enseñanzas universitarias oficiales. Dicha ley, en su preámbulo, expresa lo siguiente: 
"Finalmente, se debe tener en cuenta que la formación en cualquier actividad profesional debe contribuir al conocimiento y desarrollo... de accesibilidad universal y diseño para todos.”

Queda clara, por lo tanto, la necesidad de incluir formación específica en las nuevas titulaciones sobre competencias o contenidos que estén relacionados directamente con la inclusión de cualquier persona en la sociedad, independiente de sus características, experiencias o bagajes personales. El Real Decreto va más allá y establece lo siguiente:

"5. Entre los principios generales que deberán inspirar el diseño de los nuevos títulos, los planes de estudios deberán tener en cuenta que cualquier actividad profesional debe realizarse:

...b) bajo los principios de accesibilidad universal y diseño para todos"

Por otro lado, la base europea en cuanto a la accesibilidad, antesala del concepto de diseño universal, tuvo lugar en 1988 con la puesta en marcha del programa HELIOS, Segundo Programa de acción de la Comunidad a favor de los minusválidos, de aplicación durante el cuatrienio 1988-1991 (Fernández, 2012). Como continuación de este primer programa, en el año 1996 se complementaron los estudios posteriores con el HELIOS II, denominado como Tercer programa de acción de la Comunidad a favor de los minusválidos. Los objetivos de ambos proyectos fueron promover la integración social y económica de las PcD, así como promocionar la vida independiente para este colectivo (Zolkowska, Kasior-Szerszen y Blaszkiewicz, 2002). En Europa, además de las Resoluciones ResAP(2001)1 y ResAP(2007)3, hay que tener en cuenta la red denominada Inclusive Design Curriculum Network. Dicha red publicó un documento denominado "Curriculum for training professionals in Universal Design" (Darzentas, et al., 2001). En este documento se especifica que el entrenamiento en diseño universal debería incluir 10 temas principales. Entre ellos destacan tales como (a) Diseño Universal y grupos de usuario objetivo, (b) Diseño centrado en el usuario, (c) Productos de apoyo y (d) Ética, legislación y privacidad.

La internacionalización del movimiento del diseño universal ha generado una expansión en la audiencia que demanda información sobre prácticas y precedentes (Fletcher, 2011). Probablemente uno de los estudios más recientes y de mayor interés respecto al análisis de la integración de los estudios sobre diseño universal en el currículum universitario es el trabajo de Dyer, Callanan y Fennell(2010). En este estudio los autores llevaron a cabo una revisión de las experiencias de enseñanza del diseño universal en el Trinity College. Uno de los datos interesantes del estudio de Dyer et al. (2010) tiene que ver con sus resultados sobre la variedad en los modos en los que se enseña el diseño universal. (Véase Tabla 1).

En 2002, Kenning y Ryhl llevaron a cabo una revisión de ejemplos de enseñanza de diseño universal en distintas escuelas de arquitectura de todo el mundo. En su revisión se incluyeron países como Australia, Bélgica, Dinamarca, Francia, Grecia, Irlanda, Japón, Noruega, Polonia, Reino Unido y E.E.U.U. A pesar de las diferencias políticas, educativas y culturales entre todos esos países, Kenning y Ryhl (2002) clasificaron las iniciativas para incorporar el diseño universal en los estudios de arquitectura en dos categorías, (a) Iniciativas para incorporar el diseño universal llevadas a cabo por un individuo o por un grupo de profesores dentro de una facultad en concreto y (b) Iniciativas surgidas a partir de proyectos piloto o de investigación a gran escala. En su revisión, concluyeron que la probabilidad de éxito en la integración del diseño universal en los estudios de arquitectura es mayor cuando la iniciativa forma parte de un proyecto mayor, y en especial cuando ese proyecto llevaba en 
funcionamiento varios años. Otro ejemplo de investigación en la que se analizan asuntos de interés relacionados con la integración en el currículo del diseño universal, es el reciente trabajo de Afacan (2011) en el que se exploran las actitudes hacia el diseño universal de profesores y estudiantes en un Departamento de Arquitectura de Interiores y Diseño del Entorno. Los resultados de este trabajo indican que la aproximación al diseño universal de profesores y estudiantes está influida por diversos factores que tienen que ver, tanto con el modo en el que se integra el diseño universal en el currículo, como con las propias características de los estudiantes.

Tabla 1. Áreas temáticas, técnicas de diseño y estrategias docentes en la enseñanza del diseño universal (adaptado de Dyer et al., 2010)

\begin{tabular}{|c|c|c|}
\hline Áreas temáticas & Técnicas de Diseño & Estrategias Docentes \\
\hline $\begin{array}{l}\text { • Diversidad } \\
\text { humana } \\
\text { - } \quad \text { Reconocimiento } \\
\text { de las múltiples formas } \\
\text { de identidad } \\
\text { - Diseño de la Vida } \\
\text { Cotidiana } \\
\text { - } \quad \text { Relaciones } \\
\text { persona-entorno } \\
\text { - Capacidades } \\
\text { Humanas (capacidades } \\
\text { sensoriales, } \\
\text { capacidades cognitivas, } \\
\text { capacidades } \\
\text { psicomotoras) } \\
\text { - Forma y } \\
\text { Dimensiones Humanas } \\
\text { - } \quad \text { Ergonomía } \\
\text { - } \quad \text { Antropometría } \\
\text { • Diseño a lo largo } \\
\text { del ciclo vital }\end{array}$ & 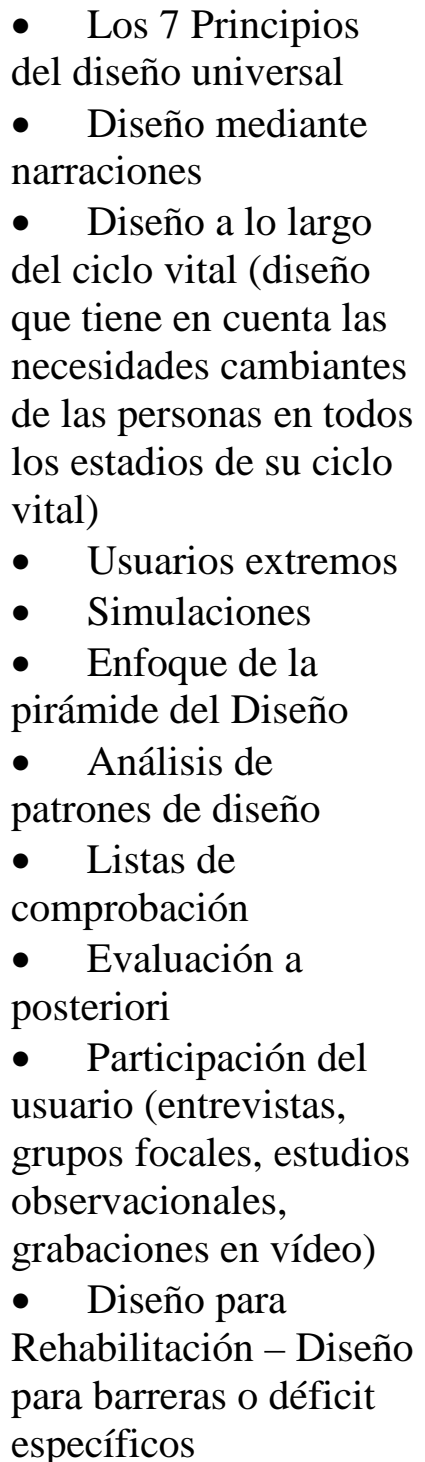 & $\begin{array}{l}\text { - } \quad \text { Clase magistral } \\
\text { - } \quad \text { Taller } \\
\text { (Presentaciones con } \\
\text { debate) } \\
\text { • Proyecto (Trabajo } \\
\text { dirigido por el } \\
\text { estudiante) } \\
\text { - Estudio de Diseño } \\
\text { (Proyectos dirigidos por } \\
\text { el estudiante con } \\
\text { espacios y equipos de } \\
\text { apoyo) } \\
\text { • Implicación de } \\
\text { agentes externos } \\
\text { (miembros de la } \\
\text { comunidad -personas } \\
\text { mayores, niños, } \\
\text { personas con } \\
\text { discapacidad-; } \\
\text { Profesionales -externos } \\
\text { que asesoran y valoran } \\
\text { críticamente los } \\
\text { proyectos del } \\
\text { estudiante-) } \\
\text { - Estrategias } \\
\text { variadas (simulaciones, } \\
\text { tutorías, uso de recursos } \\
\text { multimedia) }\end{array}$ \\
\hline
\end{tabular}

Un trabajo importante que cabe destacar es publicado por Welch (1995), o el más reciente editado por Christophersen (2002), Universal Design: 17 ways of thinking and teaching. En este libro, Christophersen compila 17 capítulos, que resumen distintas experiencias sobre inclusión de diseño universal en escuelas de arquitectura de diferentes 
países de Europa, Norte América, Asia y Australia (e.g.,Østergaard, 2002; Walker, 2002). El libro también muestra otras reflexiones y asuntos de interés. Por ejemplo, Dale y Leknes (2002) destacan el necesario carácter interdisciplinar en la formación sobre diseño universal, describiendo una experiencia de seminario compartido entre un Departamento de Terapia Ocupacional y otro de Diseño Arquitectónico. Y Yanagisawa y Shimizu (2002), muestran una experiencia de organización de un concurso de ideas como medio para promover comprensión de los estudiantes sobre el diseño universal y para ayudarles a reflexionar sobre la integración de los conceptos del diseño universal en sus propios proyectos.

En el ámbito concreto de los estudios de informática (computer sicence) destaca el trabajo de Ribu (2007). El trabajo describe como la Universidad de Oslo, dentro de una nueva titulación denominada como Informática Aplicada, y en colaboración con las organizaciones de PcD, ha desarrollado un programa educativo en el que grupos de 4-5 estudiantes desarrollaron soluciones basadas en la usabilidad para los usuarios con discapacidad.

Más recientemente, Iglesias, Saraiva y Lloredo (2011), realizan un análisis de la accesibilidad universal en las enseñanzas universitarias españolas. Los autores han identificado las asignaturas que estén dedicadas a materias relacionadas con la accesibilidad dentro de los grados en los que procede su inclusión. Según los resultados de este informe, en promedio, el porcentaje de cumplimiento de las universidades españolas es de un $16 \%$ ( $S D=31$; Min=0; Max=100) encontrando asignaturas sobre "accesibilidad" en 24 de las 76 universidades analizadas.

Por lo tanto, en base a diversos trabajos que se están llevando a cabo en entornos similares al español, este estudio tiene como objetivo principal analizar la situación española en la inclusión de contenidos sobre diseño universal en las titulaciones de ingeniería informática y en arquitectura.

\section{Método}

Selección de la población de estudio

Para seleccionar los títulos de grado que han conformado la muestra en este trabajo, se realizó una búsqueda de todos los títulos de grado de universidades españolas, públicas y privadas, de la rama de ingeniería y arquitectura y sus titulaciones afines. La búsqueda de títulos se llevó a cabo desde la página Web que da acceso al mapa de titulaciones de las universidades españolas del Ministerio de Educación, Cultura y Deporte ( https://www.educacion.gob.es/notasdecorte). La búsqueda devolvió un total de 905 títulos académicos. Estos grados corresponden a la población total de la rama de conocimiento de ingeniería y arquitectura en el estado español en el momento de la realización de la búsqueda. A partir de este listado general, finalmente, se seleccionaron 159 títulos de grado: todos los títulos de grado que estaban dentro de las temáticas relacionadas con arquitectura y sus afines, y todos los títulos grado de ingeniería informática y sus afines.

Para poder analizar de una manera comparable la información de las 159 titulaciones, se utilizaron los planes de estudio de cada uno de los títulos de grado identificados. Éstos son los documentos oficiales que contienen toda la información sobre las características de cada uno de los títulos de grado. Para realizar esta búsqueda se utilizó la página Web que el Gobierno de España pone a disposición de los ciudadanos para consulta del histórico del Boletín Oficial del 
Estado. Mediante este procedimiento de búsqueda en el BOE, se obtuvieron 108 resoluciones (67.92\% del total). Con el fin de conformar una muestra de documentos comparables, para el resto de títulos cuyos planes aún no habían sido publicados en el BOE, se buscó información en las páginas Web de aquellas universidades o facultades que habían sido identificadas en la muestra total de las 159 titulaciones.

\section{Población de estudio}

El total de 159 documentos, en los que se describe el programa general de las titulaciones relacionadas con la arquitectura y la ingeniería informática en España, fue la población final que estaba disponible en el momento de llevar a cabo este trabajo. La distribución general de los títulos seleccionados en función de las dos grandes áreas temáticas quedó conformada con un total de 47 titulaciones de Arquitectura (29.56\%), respecto de un total de 112 de Ingeniería Informática (70.4\%).Más detalladamente, la Tabla 2 muestra totales y porcentajes en función de la denominación específica de los títulos. Respecto al tipo de universidad del que procedían los títulos, un total de 44 títulos (27.67\%) estaban ubicadas en universidades privadas, frente a los 115 títulos (72.33\%) que fueron universidades públicas.

Tabla 2. Porcentaje y número de títulos por denominación de título

\begin{tabular}{ccc}
\hline Tipo título & $\mathbf{N}^{\mathbf{0}}$ de títulos & $\mathbf{\%}$ \\
\hline Arquitectura & 29 & 18.24 \\
Audiovisuales & 2 & 1.26 \\
Comunicaciones & 1 & 0.63 \\
Edificación & 18 & 11.32 \\
Información & 10 & 6.29 \\
Informática & 63 & 39.62 \\
Ingeniería de Computadores & 3 & 1.89 \\
Multimedia & 4 & 2.52 \\
Ingeniería de Sistemas & 1 & 0.63 \\
Software & 7 & 4.40 \\
Telemática & 15 & 9.43 \\
Tecnología de la Información y & 6 & 3.77 \\
la Comunicación (TIC) & & \\
\hline
\end{tabular}

\section{Procedimiento}

Para llevar a cabo la detección de las denominaciones de asignaturas dedicadas a las temáticas relacionadas con el diseño universal, se llevó a cabo un análisis de contenido sobre los documentos correspondientes a los 159 títulos seleccionados.En primer lugar, para la categorización de los 159 títulos de grado se utilizaron diversos criterios en la nomenclatura de los documentos que sirvieron para otorgar un nombre específico a cada documento. Se definió un sistema de codificación para la denominación de los documentos que reflejara un 
identificador único, el tipo de universidad, pública o privada, la comunidad autónoma y la temática del título.

En segundo lugar, se procedió a identificar dentro de cada uno de los títulos aquellas denominaciones de asignaturas que por su temática deberían desarrollar contenidos y competencias relacionadas con el diseño universal, diseño para todos o accesibilidad universal. El proceso de detección de las denominaciones de asignaturas que pudieran tratar sobre diseño universal o diseño para todos consistió en la búsqueda, en cada documento, de asignaturas que en su denominación incluyeran algún término relacionado con el diseño universal o el diseño para todos en base a una serie de términos relacionados. La lista de términos relacionados con el diseño universal se generó a partir de la revisión de términos utilizados en otros estudios (Dyer et al., 2010; Ribu, 2007). En concreto, los términos utilizados fueron los siguientes:

Accesibilidad, Acceso universal, Adaptación (adaptadas, adaptado), Discapacidad, Diseño accesible, Diseño sin barreras , diseño inclusivo, diseño para todos , diseño universal, Diversidad, Domótica, Ergonomía, Hombre, Hombre-máquina, Humano, Igualdad, Inclusión, Inclusivo, Integración, Interacción, Normalización, Participación, Persona, Persona-ordenador, Rehabilitación, Sociedad, Universal (universalidad), Usabilidad, Usuario/os.

\section{Análisis de datos}

Cada uno de los términos identificados fue utilizado en un proceso de búsqueda iterativo sobre la base de documentos primarios. El proceso de búsqueda se llevó a cabo mediante el software de análisis cualitativo ATLAS.Ti. Ésta es una herramienta informática cuyo objetivo es facilitar el análisis de, principalmente, grandes volúmenes de datos textuales (Muñoz, 2003). Con el uso del ATLAS.Ti, se realizó la categorización de aquellas titulaciones que contenían alguno de los términos anteriores y que quedaron identificadas como titulaciones que podrían enseñar algún tipo de contenidos relacionados con el diseño universal o diseño para todos en las titulaciones de arquitectura e ingeniería informática.

\section{Resultados}

El proceso de búsqueda condujo a la identificación de un total de 79 coincidencias en títulos de asignaturas con los términos utilizados en la búsqueda iterativa. La Tabla 3 muestra la frecuencia total de cada uno de los términos encontrados.

Como primer resultado cabe destacar la escasa presencia de términos relacionados con el diseño universal o diseño para todos de manera genérica en la denominación de las asignaturas. Además, hay que tener en cuenta que la tabla anterior expresa una frecuencia individual lo que significa que algunos descriptores podían estar contenidos en un único título de una asignatura. Éste es el ejemplo de interacción, que por ejemplo, se identificó en asignaturas con el título de Interacción hombre-máquina o Interacción persona-ordenador.

Tabla 3. Términos en denominaciones de asignaturas por frecuencia (algunas asignaturas contenían más de un término)

\begin{tabular}{cc}
\hline Término & Frecuencia \\
\hline Sociedad & 19 \\
Interacción & 18
\end{tabular}




\begin{tabular}{cc} 
Persona-ordenador & 11 \\
Accesibilidad & 9 \\
Usabilidad & 9 \\
Domótica & 8 \\
Diseño & 5 \\
Adaptación & 3 \\
Ergonomía & 2 \\
Hombre-Máquina & 2 \\
Universalidad & 2 \\
Contexto & 1 \\
Factores Humanos & 1 \\
\hline
\end{tabular}

Un segundo resultado de interés, es la descripción de aquellos términos para los que no se obtuvo ninguna coincidencia en la denominación de las asignaturas de los 159 títulos analizados. En concreto aquellos descriptores que obtuvieron una frecuencia igual a cero fueron:

Acceso universal, Discapacidad, Diseño accesible, Diseño sin barreras, diseño inclusivo, diseño para todos, diseño universal, Diversidad, Igualdad, Inclusión, Inclusivo, Integración, Necesidades, Normalización, Participación, Política, Políticas, Psicología, Rehabilitación.

Tabla 4. Frecuencias totales de las titulaciones que contienen denominaciones de asignaturas relacionadas con el diseño universal

Título $\quad$ Frecuencia

\begin{tabular}{lc}
\hline $\begin{array}{l}\text { Arquitectura } \\
\text { (N total de títulos = 9) }\end{array}$ & \\
\hline Edificación & 5 \\
Arquitectura & 4 \\
\hline $\begin{array}{l}\text { Informática y Tecnologías de la } \\
\text { Información y la Comunicación } \\
\text { (N total de títulos = 49) }\end{array}$ \\
\hline Ingeniería Informática & \\
Ingeniería Telemática & 25 \\
Ingeniería de Software & 6 \\
Tecnologías de la Información y la & 6 \\
Comunicación & 4 \\
Ingeniería Multimedia & 3 \\
Ingeniería de Computadores & 2 \\
Ingeniería Sistemas de Información & 2 \\
Ingeniería de Sistemas Audiovisuales & 1 \\
\hline \multicolumn{2}{c}{ Total } \\
\hline
\end{tabular}

Si el análisis se centra en los resultados del total de universidades, un total de 33 (45.83\%), de una población de 72 universidades dispondrían de alguna denominación de materia que ha sido identificada con los descriptores relacionados con el diseño universal o diseño para todos. Teniendo en cuenta la titularidad de la universidad el resultado obtenido fue 
de un total de 23 universidades públicas (71.87\%), frente a 10 universidades privadas (28.13\%).

Por otro lado, un total de 58 titulaciones de grado fueron identificadas en el análisis realizado. En concreto, en la Tabla 4 se pueden observar los resultados del número de titulaciones con sus respectivas denominaciones y las frecuencias totales, divididas en las áreas de conocimiento.

Otro resultado que resulta interesante destacar, es la diferencia existente entre los totales de las titulaciones de ingeniería informática y arquitectura, así como los porcentajes relativos del total de titulaciones que se identificaron en la muestra de cada una de las dos áreas temáticas (Véase Tabla 5).

Tabla 5. Titulaciones divididas en las áreas de ingeniería informática y de arquitectura

\begin{tabular}{cccc}
\hline & Total & $\begin{array}{c}\text { \% } \\
\text { total }\end{array}$ & $\begin{array}{c}\text { \% } \\
\text { relativo }\end{array}$ \\
\hline Ingeniería Informática & 49 & 84.48 & 43.75 \\
Arquitectura & 9 & 15.52 & 19.14 \\
\hline
\end{tabular}

Finalmente, se calculó la frecuencia de los términos de búsqueda. Los totales muestran los términos con mayores frecuencias sociedad e interacción, ambos presentes en 19 y 18 denominaciones de asignaturas. De especial atención para este trabajo, fueron los resultados relacionados con los términos relacionados directamente con la accesibilidad y usabilidad. En concreto, se encontraron 9 registros para cada uno de los dos términos.

\section{Discusión}

Este trabajo tenía como objetivo principal analizar el mapa de titulaciones de arquitectura e ingeniería informática del sistema universitario español para obtener información sobre la inclusión de contenidos y competencias relacionadas con el diseño universal.Los resultados del análisis de contenido, nos permiten estimar que solo se han encontrado 65 denominaciones de asignaturas en 159 títulos distintos de grado, con lo que se puede concluir que ni siquiera la mitad de los títulos de grado tendrían en cuenta lo establecido por el Real decreto 1393/2007, en cuanto a la inclusión de contenidos relacionados con la accesibilidad universal o diseño para todos. Solo 33 universidades se han identificado como instituciones en las que existen denominaciones de asignaturas relacionadas con el diseño universal. Este dato es algo superior respecto a la estimación del estudio llevado a cabo por Iglesias et al. (2011), en el que el número total de universidades encontradas con denominaciones de asignaturas fue de 22. La diferencia, seguramente, es debida a los términos de búsqueda utilizados por cada estudio. En este estudio la selección de términos se realizó desde una perspectiva más amplia y considerando al diseño universal como un paradigma que abarca un número más elevado de ámbitos, mientras que en el estudio de Iglesias et al., el enfoque era mas reduccionista y centraron su búsqueda en el término "accesibilidad."

Como resultado más destacable en cuanto al número de titulaciones encontradas en este estudio, es necesario describir la diferenciación que existe entre el número total de titulaciones 
con posibles enseñanzas sobre diseño universal respecto de las dos áreas seleccionadas para este estudio. Solo 9 titulaciones del área de arquitectura han sido descritas como posibles grados donde se imparte contenidos relacionados con el diseño universal o diseño para todos, frente a las 49 titulaciones del área de ingeniería informática donde se están llevando a cabo las enseñanzas acerca de este paradigma. Éste es un resultado importante de destacar, ya que en las titulaciones de arquitectura, donde cabría esperar una presencia de manera clara e inequívoca de términos relacionados con el diseño para todos o accesibilidad universal, tiene una escasa frecuencia.

Por otro lado, si atendemos a las denominaciones de asignaturas en las que se ha encontrado referencias al diseño universal o a concepciones similares, como aspecto positivo cabe destacar que las temáticas encontradas, son coincidentes que con las áreas identificadas por otros autores (Darzentas et al., 2001; Dyer et al., 2010).Sin embargo, como aspecto negativo habría que hacer referencia a la nula presencia de un concepto como el de diseño para todos o accesibilidad universal en la denominación de asignaturas, presente en el propio Real Decreto que regula las enseñanzas universitaria. Esta terminología, que además se justifica desde la propia ley de atención a las PcD en España y que es la más extendida en nuestro país, no tiene, al menos en el momento de realizarse el análisis de contenido, un reflejo dentro de los títulos de grado de arquitectura e ingeniería informática.

A modo de resumen, hay que destacar que el análisis del conjunto de las titulaciones de arquitectura e ingeniería informática en nuestro país, no hace más que describir una situación de incumplimiento, al menos parcial ya que además se podría considerar también la necesidad de evaluar los máster ofertados por las universidades españolas, en relación a la enseñanza del diseño para todos o accesibilidad universal.

Finalmente, se considera importar matizar que este trabajo se ha mantenido en nivel de análisis basado en denominaciones de asignatura y que, por tanto, deben valorarse los resultados como lo que son. Debido a las dificultades encontradas para obtener un documento homogéneo en las páginas Web institucionales de todas las universidades del país, no ha sido posible realizar un análisis del contenido de cada una de las asignaturas.

\section{Referencias}

Afacan, Y. (2011). Teaching universal design: an empirical research in interior architecture. Procedia - Social and Behavioral Sciences, 15, 3185-3192. doi:10.1016/j.sbspro.2011.04.269

Christophersen, J. (Ed.). (2002). Universal Design: 17 Ways of Thinking and Teaching. Norway: Husbanken.

Consejo de Europa. (2001, February 15). Resolución ResAP (2001)1 sobre la introducción de los principios de diseño universal en los curricula de todas las actividades relacionadas con el entorno de la construcción. Resolución. Extraído el 10 de Febrero de 2013 desde http://www.ceapat.es/InterPresent2/groups/imserso/documents/binario/im_029652.htm

Consejo de Europa. (2007). Resolución ResAP (2007)3 “Alcanzar la plena participación a través del Diseño Universal.” Resolución. Extraído el 10 de Febrero de 2013 desde http://www.ceapat.es/InterPresent1/groups/imserso/documents/binario/200808010002_4 _4_0-4.pdf 
Dale, S., y Leknes, R. (2002). Universal Design - an Interdisciplinary Challenge. En J. Christophersen (Ed.): Universal Design: 17 Ways of Thinking and Teaching (pp. 59-80). Norway: Husbanken.

Darzentas, J., Colette, N., Romero, R., Engelen, J., Strobbe, C., Velasco, C. A., Petrie, H. (2001). D3.2 - Identifying Core Knowledge and Skill Sets for Model Curricula: update (Public Report No. IST-2001-38786). Extraído el 14 Febrero de 2013desde http://webcc.fit.fraunhofer.de/downloads/projects/IDCnet/IDCnet_D3.2.1.pdf

Dyer, M., Callanan, M., y Fennell, A. (2010). Integrating Universal Design Content in Third Level Curriculum. National Disability Authority, Centre for Excellence in Universal Design, James Hubbard.

Fernández, M.T. (2012). El diseño universal: concepto y certificación. Boletín del Real Patronato Sobre Discapacidad, 75, 4-10.

Fletcher, V. (2011).An international web-based collection of universal design exemplars. En W. Preiser y K.H. Smith (Eds.): Universal Design Handbook Second Edition. New York: McGraw Hill.

HELIOS (1988). Segundo Programa de Acción de la Comunidad a favor de los minusválidos. Bruselas, Bélgica: European Commission. Extraído el 10 de Marzo de 2013 desde http://ec.europa.eu/education/higher-education/publ_en.htm

HELIOS II (1996). Tercer programa de acción de la Comunidad a favor de los minusválidos. Bruselas, Bélgica: European Commission. Extraído el 10 de Marzo de 2013 desde http://ec.europa.eu/education/higher-education/publ_en.htm

Iglesias, A., Saraiva, G., y Lloredo, L. (2011). Informe sobre la presencia de la accesibilidad universal en las enseñanzas universitarias: Sobre la aplicación del artículo 3.5 del Real Decreto 1393/2007, de 29 de octubre, por el que se establece la Ordenación de la Enseñanzas Universitarias Oficiales (No. 17). Madrid: Clínica Jurídica "La enseñanza de los derechos fundamentales en la universidad española" del Instituto de derechos humanos Bartolomé de las Casas de la Universidad Carlos III de Madrid. Extraído el 03 de Abril de 2013 desde http://www.tiempodelosderechos.es/docs/oct11/informe17.pdf

Kennig, B., y Ryhl, C. (2002). Teaching Universal Design: Global Examples of Projects and Models for Teaching in Universal Design at Schools of Design and Architecture. AAOutils. Extraído el 03 de Abril de 2013 desde www.anlh.be/aaoutils/aaoutils/examples.PDF

LEY 51/2003, de 2 de diciembre, de igualdad de oportunidades, no discriminación y accesibilidad universal de las personas con discapacidad persona (2 de Diciembre).

LEY ORGÁNICA 2/2006, de 3 de mayo, de Educación. (2006).

LEY ORGÁNICA 4/2007, de 12 de abril, por la que se modifica la Ley Orgánica 6/2001, de 21 de diciembre, de Universidades. (2007).

Muñoz, J. (2003). Análisis cualitativos de datos textuales con ATLAS/ti. Extraído el 15 de Junio de 2013 desde http://www.ugr.es/ textinfor/documentos/manualatlas.pdf

Østergaard, P. (2002). Architects of Tomorrow, Accessibility of the Future - Teaching Accessibility at the School of Architecture. En J. Christophersen (Ed.): Universal Design: 17 Ways of Thinking and Teaching, (pp. 149-164). Norway: Husbanken. 
Preiser, W. (2011). Toward Universal Design Performance Assessments. En W. Preiser y K.H. Smith (Eds.): Universal Design Handbook Second Edition. (pp. 210-217) New York: McGraw Hill.

Real Decreto 1393/2007, de 29 de octubre, por el que se establece la ordenación de las enseñanzas universitarias oficiales. (2007).

Ribu, K., (2007). Introducing Usability Engineering and Universal Design in the Computer Science Curriculum. International Conference on Engineering Education \& Research.

Walker, A. (2002). Developments Towards Inclusive Design Teaching. En J. Christophersen (Ed.): Universal Design: 17 Ways of Thinking and Teaching, (pp. 127-148). Norway: Husbanken.

Welch, P. (1995). Strategies for Teaching Universal Design. Boston: Adaptive Environments. Yanagisawa, M., y Shimizu, T. (2002).Universal Design Competition for Students 2000. En J. Christophersen (Ed.): Universal Design: 17 Ways of Thinking and Teaching (pp. 373386). Norway: Husbanken.

Zolkowska, T., Kasior-Szerszen, I., y Blaszkiewicz, I. (2002). European Union Policy Toward People with Disabilities. DisabilityStudiesQuarterly, 22(4), 217-224. 